\title{
Quels critères pour l'examen de spécialiste? (Etat 2010)
}

\author{
L'Institut suisse pour la formation médicale postgraduée et continue (ISFM) introduit \\ la clause éliminatoire pour l'examen de spécialiste en néphrologie ainsi que pour les \\ examens de formation approfondie en pathologie moléculaire et en gastroentérolo- \\ gie et hépatologie pédiatrique.
}

\section{Christoph Hänggeli}

Administrateur responsable de l'Institut suisse pour la formation médicale postgraduée et continue (ISFM)
Correspondance: Christoph Hänggeli ISFM/FMH Elfenstrasse 18 $\mathrm{CH}-3000$ Berne 15 Tél. 0313591111 Fax 0313591112 siwf@fmh.ch

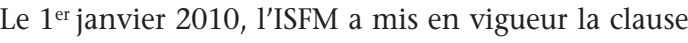
éliminatoire de l'examen de formation approfondie pour deux nouvelles spécialités et, rétroactivement au $1^{\text {er }}$ janvier 2009 pour l'examen de spécialiste en néphrologie. Ainsi, la réussite de l'examen de spécialiste ou de la formation approfondie est désormais exigée pour tous les titres postgrades fédéraux (à l'exception de la chirurgie maxillo-faciale) et pour une partie des formations approfondies, sous réserve des dispositions transitoires applicables.

\section{Dispositions transitoires}

Font exception les candidats remplissant une des deux conditions suivantes:

- Les candidats ayant déjà participé (entièrement ou en partie) à un examen de spécialiste ou un examen de formation approfondie dans le domaine concerné avant l'entrée en vigueur du caractère éliminatoire de l'examen ne devront pas passer d'autre examen.

- Les candidats terminant leur formation postgraduée dans les deux ans après l'entrée en vigueur du caractère éliminatoire de l'examen de spécialiste ne doivent attester, pour l'obtention du titre ou de la formation approfondie, que leur participation à l'examen. Attention: toutes les conditions doivent être remplies durant les deux ans qui suivent la date d'entrée en vigueur (y compris l'examen de spécialiste ou l'examen de formation approfondie complet, sauf si la société de discipline médicale refuse la participation à la $2^{\mathrm{e}}$ partie de l'examen en raison d'un résultat insuffisant à la $1^{\text {re }}$ partie)!

Pour les disciplines ci-après, seule la participation à l'examen continue d'être exigée, en d'autres termes: il n'est pas nécessaire d'avoir réussi l'examen pour obtenir le titre de spécialiste.

- Chirurgie de la main

- Chirurgie maxillo-faciale

- Neuropathologie

\section{Tableau}

Dois-je réussir l'examen de spécialiste ou de formation approfondie? (suite à la page suivante)

Titres de spécialiste

Allergologie et immunologie clinique

1. 1.2003

Anesthésiologie

Angiologie

Cardiologie

4. 1986

Chirurgie (y c. examen de base)

1.2005

1. 1.1999

Chirurgie cardiaque et vasculaire thoracique

1.1 .1999

Chirurgie de la main

1.2008

Chirurgie maxillo-faciale

1.1.2011?

Chirurgie plastique, reconstructive et esthétique

Chirurgie orthopédique

1.1.2011?

1. 1.2004

Chirurgie pédiatrique

1. 1.2003

Dermatologie et vénéréologie

2. 4.1986

Endocrinologie/diabétologie

1.1.2003

Gastroentérologie

Génétique médicale
1.1.2001

1. 1.2000

1.1.2008
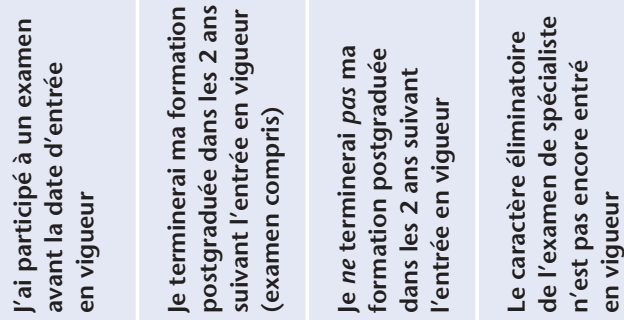


\section{Tableau}

Dois-je réussir l'examen de spécialiste ou de formation approfondie? (suite de la page précédente)

Titres de spécialiste

Gynécologie et obstétrique

1.1 .1999

Hématologie

Infectiologie

1. 1.2001

Médecine du travail

1. 1.2003

Médecine générale

1. 1.2001

Médecine intensive

1. 1.2000

Médecine interne

1. 1.2001

Médecine légale

1. 1.1999

Médecine nucléaire

1.1.2001

Médecine pharmaceutique

4.1986

Médecine physique et réadaptation

1. 1.2003

Médecine tropicale

Néphrologie

Neurochirurgie

Neurologie

Neuropathologie

1.1.2003

1.1. 2001

1.1.2009

2. 4.1986

1. 1.2003

1.1.2011?

Oncologie médicale

1. 1.2001

Ophtalmologie

ORL (sans la chirurgie maxillo-faciale)

Pathologie

Pharmacologie et toxicologie cliniques

1. 1.2002

1. 1.2001

1.1 .1999

Pédiatrie

Pneumologie

Prévention et santé publique

Psychiatrie et psychothérapie

Psychiatrie et psychothérapie

d'enfants et d'adolescents

Radiologie

1.1.2006

1.1.2004

1.1. 2003

1.1.2004

1. 1.2001

1. 1.2002

2. 4.1986

Radio-oncologie / radiothérapie

2. 4.1986

Rhumatologie

Urologie

1. 1.2001

1.1. 2002

Formations approfondies

Chirurgie générale et d'urgence

1.1.2009

Chirurgie thoracique

1.1.2007

Chirurgie vasculaire

1.1.2007

Chirurgie viscérale

Endocrinologie-diabétologie pédiatrique

1. 1.2009

(avec les dates actuelles

d'examen) se trouve sur le site internet de la $\mathrm{FMH}$ à I'adresse suivante: www. siwf.ch. Les dates d'examen sont aussi publiées dans le Bulletin des médecins suisses (BMS). Pour de plus amples informations, veuillez vous adresser à I'Institut suisse pour la formation médicale postgraduée et continue (ISFM), case postale 170, Elfenstrasse 18, 3000 Berne 15, tél. 031359 11 11, fax 03135911 12, courriel: siwf@fmh.ch

Gastroentérologie et hépatologie pédiatrique

1.1.2005

Cardiologie pédiatrique

1.1.2010

1.1. 2001

Gériatrie

1.1.2006

1.1.2003

Pathologie moléculaire

1. 1.2010

Neuropédiatrie

1.1.2003

Urologie opératoire

1.1. 2009

Phoniatrie

1.1.2007

Pneumologie pédiatrique

1.1. 2009

Radiologie pédiatrique

1.1.2006

toutes les autres formations approfondies

$\mathrm{R}=$ Réussite; $\mathrm{P}=$ Participation
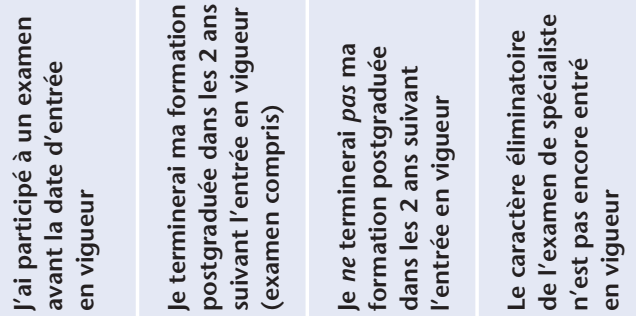

Néonatologie 